\title{
Next Generation Reservoir Computing
}

\author{
MLIK SALWA ${ }^{1}$ \\ ${ }^{1}$ Affiliation not available
}

November 11, 2021

\section{Introduction:}

L'article «Next generation reservoir computing » est un article récent publié en 21 septembre 2021 dans le journal à accès libre «Nature communication ». Il a pour référence et identifiant sont Nat Commun 12, 5564 (2021) ; DOI: 10.1038/s41467-021-25801-2. Les contributeurs de cet article sont :

Daniel J. Gauthier: Un professeur de physique à l'Université d'État de l'Ohio, aux États-Unis. Il a aussi contribué en plus de 200 articles dans différents domaines scientifiques.

Erik Bollt: Un professeur au sein de l'université de Clarkson aux États-Unis, Département d'ingénierie électrique et informatique. Aussi c'est le directeur du centre de science des systèmes complexe du Clarkson, Il est contributeur dans plus de 200 articles scientifiques.

Aaron Griffith: Lauréat de l'Université d'état de l'Ohio, aux États-Unis. Ses 3 d'autres contributions dans la recherche traitent aussi le réservoir computing.

Wendson A. S. Barbosa: Un chercheur au sein du département de physique à Université d'État de l'Ohio, aux États-Unis, il est contributeur dans 4 autres articles dont un est aussi sur le réservoir computing.

Par ce présent rapport nous allons en premier temps discuter le contexte de cet article et son positionnement par rapport à l'existant. Après nous allons voir les contributions apportées par les auteurs et qu'est-ce qu'ils ont trouvé comme résultats après leur recherche.

\section{Contexte de l'article}

Le contexte de cet article est la prédiction des systèmes dynamiques. Un système dynamique est un système qui évolue au cours du temps. Nous cherchons toujours à prévoir cette évolution afin de savoir le comportement futur du système en se basant sur ses états précédemment observés. Comme exemple : la prédiction du système météorologique de la terre. Les méthodes et les approches récentes du Machine learning nous génèrent des modèles à travers lesquels nous obtenons ces prévisions.

Le paradigme du ML (Machine learning) le plus adapté à ce type de système est le Réservoir Computing $\neg$ RC. Il traite les données chronologiques observées et nous donne une estimation d'état futur, même dans les systèmes les plus complexes et difficiles. Ici dans cet article l'auteur lance la nouvelle génération de ce Réservoir Computing. 


\section{Positionnement par rapport à l'existant}

Le Réservoir Computing traditionnel est basé sur le réseau neuronal récurrent dont les neurones sont interconnectés. Il est efficace dans la prévision des systèmes dynamiques, sa force vient de son pouvoir calculer et se prêter très bien la descente du gradient ce qui permet d'ajuster les paramètres de ses neurones pour mieux expliquer les données.

La figure suivante montre la prédiction du Réservoir computing traditionnel appliquée sur le cas de lorenz en temps discret.

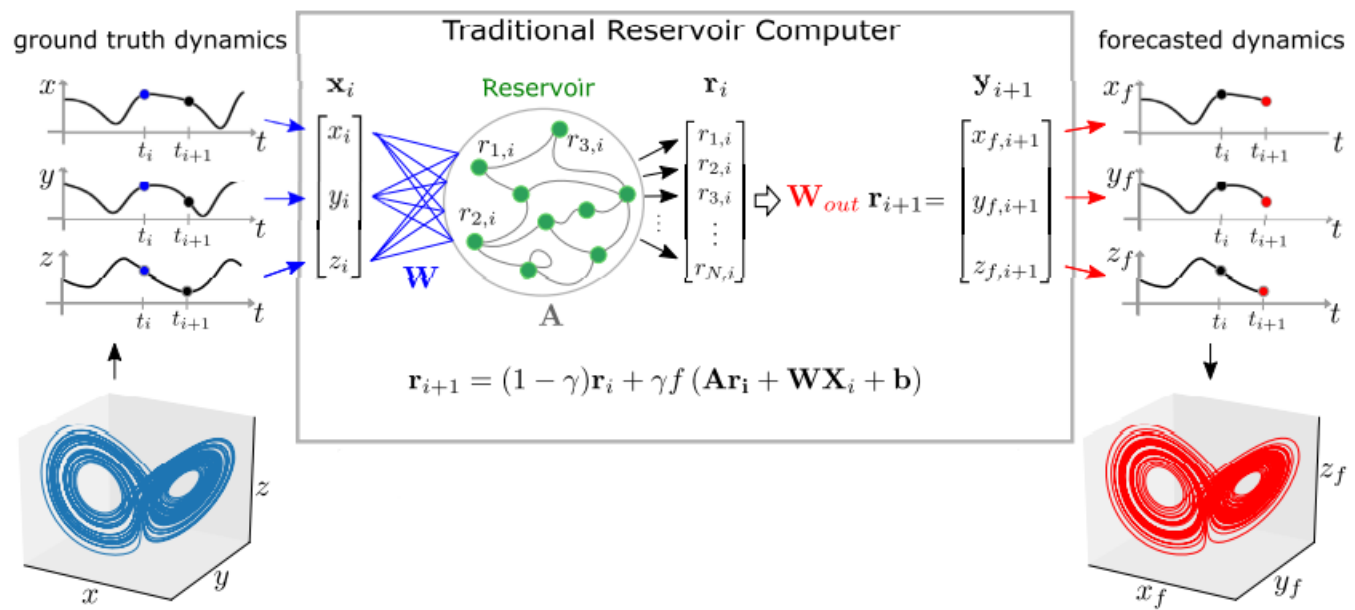

Figure 1: Traditional Reservoir Computing

Comme illustre cette figure, on diffuse des données d'entrée, en affectant un coefficient $\boldsymbol{W}$ à choisir aléatoirement mais reste fixe, dans un réservoir de grande dimension composé de $\mathrm{N}$ nœuds interconnectés par la matrice de connectivité $\boldsymbol{A}$ qui est aussi choisie aléatoirement mais ne change pas. On présente la dynamique de ce système par :

$$
\mathbf{r}_{i+1}=(1-\gamma) \mathbf{r}_{i}+\gamma f\left(\mathbf{A r}_{i}+\mathbf{W X} \mathbf{X}_{i}+\mathbf{b}\right)
$$

or $\boldsymbol{r}_{\boldsymbol{i}}$ est le vecteur qui présente l'état de chaque nœud au point du temps $\boldsymbol{t}=\boldsymbol{i}$ et $\boldsymbol{r}_{\boldsymbol{i}+\boldsymbol{1}}$ est l'état du réservoir au point suivante $\boldsymbol{t}=\boldsymbol{i}+\boldsymbol{1}$.

$\boldsymbol{f}$ est la fonction d'activation appliqué sur les composantes vectorielles et $\boldsymbol{b}$ est le vecteur de biais de noud.

$\boldsymbol{g}$ c'est le decay rate des nœuds ( Exemple: si $\mathbf{i}$ est extrêmement proche de $\mathbf{i}+\mathbf{1}$ alors g tend vers alors $\boldsymbol{r}_{\boldsymbol{i}+\boldsymbol{1}}$ sera égale à $\boldsymbol{r}_{\boldsymbol{i}}$ ).

Pour prédire donc $\boldsymbol{Y}_{\boldsymbol{i}+\boldsymbol{1}}$ on utilise $\boldsymbol{W}_{\text {out }}$ la matrice des poids de sortie, cette matrice est obtenue grâce à l'entrainement en donnant les entrées $\boldsymbol{X}_{\boldsymbol{i}}$ et la sortie désirée $\boldsymbol{Y}_{\boldsymbol{d}}$ par la relation suivante :

$$
\mathbf{W}_{\text {out }}=\mathbf{Y}_{d} \mathrm{O}_{\text {total }}{ }^{T}\left(\mathrm{O}_{\text {total }} \mathrm{O}_{\text {total }}{ }^{T}+\alpha \mathbf{I}\right)^{-1}
$$


Ou la deuxième expression présente la pseudo-inverse du $\mathbf{O}_{\text {total }}$ avec $\boldsymbol{a}$ paramétre pour rendre $\mathrm{W}_{\text {out }}$ stable.

Donc $\boldsymbol{Y}_{i+1}=\boldsymbol{W}_{\text {out }} \boldsymbol{O}_{\text {total,i+1 }}$; cette expression $\mathbf{O}_{\text {total,i+1 }}$ juste pour dire qu'il peut être linéaire comme non linéaire mais dans cette approche $\boldsymbol{O}_{\text {total, },+1}$ est linéaire qui est $\boldsymbol{r}_{i+1}: \boldsymbol{O}_{\text {total }, i+1}=\boldsymbol{O}_{\text {lin, }, i+1}=\boldsymbol{r}_{i+1}$

$$
\text { Alors : } \quad \boldsymbol{Y}_{i+1}=\boldsymbol{W}_{\text {out }} \mathrm{ri+1}
$$

NB: Dans cette approche on adopte une fonction d'activation Non linéaire et un vecteur caractéristique linéaire

\section{Contributions}

La méthode du Réservoirs Computing traditionnelle est une méthode puissante pour prédire le système dynamique. Mais elle présente certains inconvénients. Comme nous avons dit dans ce qui précédent, Les matrices $\boldsymbol{A}$ et $\boldsymbol{W}$ sont des métaparamètres qui sont choisis aléatoirement donc nous ne pouvons pas être sure de leurs performances. Peut-être nous aurons de la chance de choisir les bonnes matrices comme nous pouvons choisir les mauvaises. Cela rendre ce choix plus complexe. Aussi le RC traditionnel nécessite de disposer une grande quantité de données.

L'auteur a constaté que cette méthode existante du réservoirs computing est égale au modèle d'autorégression vectorielle non linéaire en mathématiques. Ainsi, il a proposé une structure simple selon les modèles d'autorégression vectorielle non linéaire, ce qui correspond à la prochaine génération du Réservoir Com-

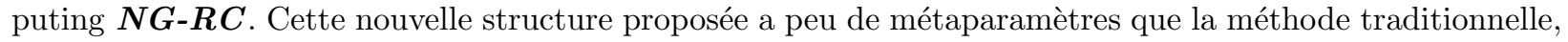
et la phase initiale de l'entrainement ne nécessite que peu de données . La figure suivante illustre cette contribution appliquée de Lorenz ( prévision de la dynamique à court terme): 


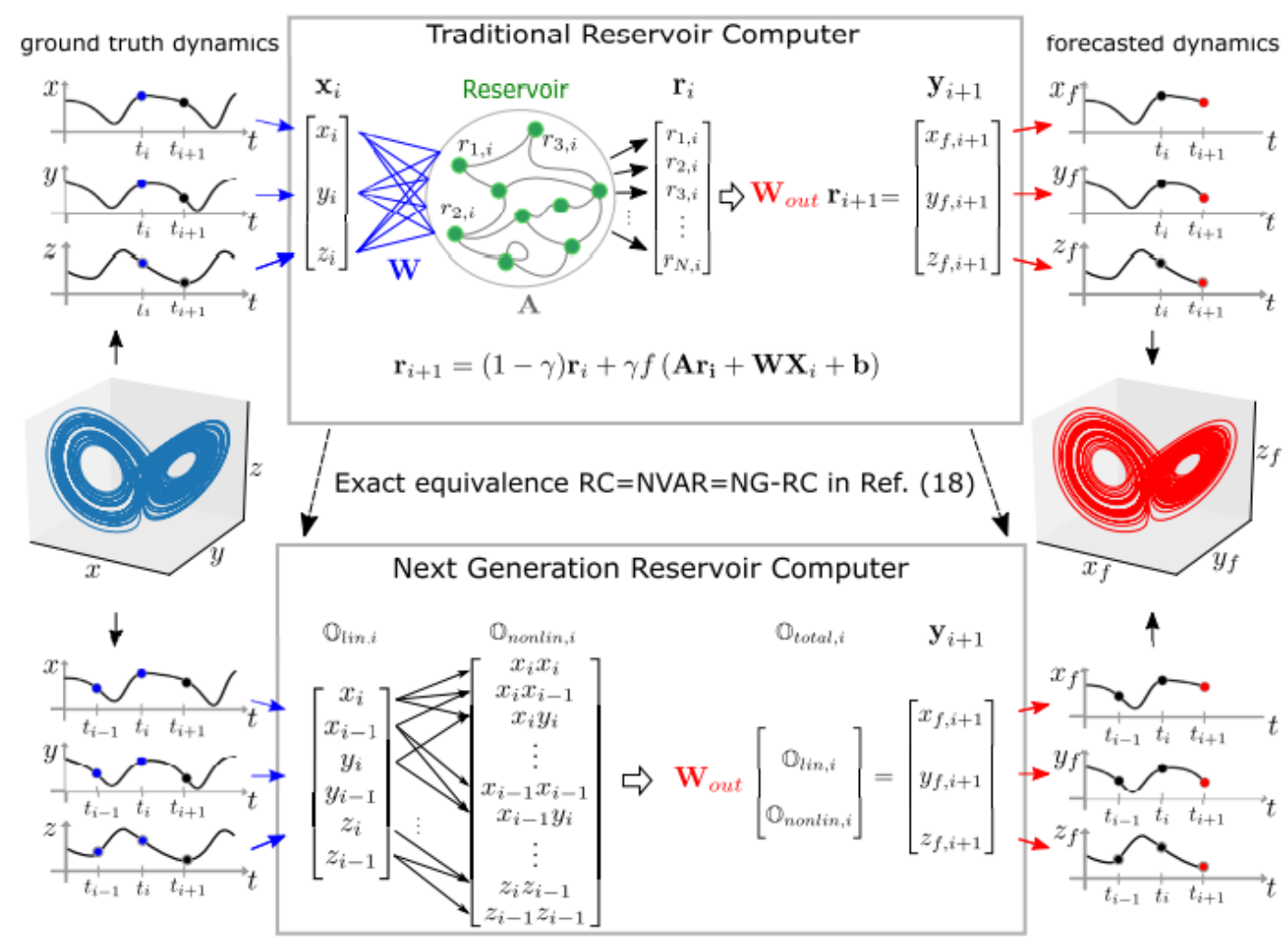

Figure 2: New Generation Reservoir Computing

\section{Les expériences réalisées et résultats}

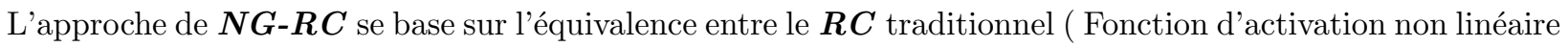
et vecteur caractéristique linéaire) et la méthode $\boldsymbol{N} \boldsymbol{V A R}$ ( Autorégression Vectorielle Non linéaire) avec une activation linéaire et vecteur caractéristique non linéaire.

Cette approche consiste alors à déplacer la non-linéarité du réservoir vers le vecteur de sortie. Donc nous n'aurons plus besoin du réservoir avec des neurones, ce qu'il veut dire la disparition des métaparamètres $\boldsymbol{W}$ et $\boldsymbol{A}$. La figure suivante illustre le concept du NG-RC: 


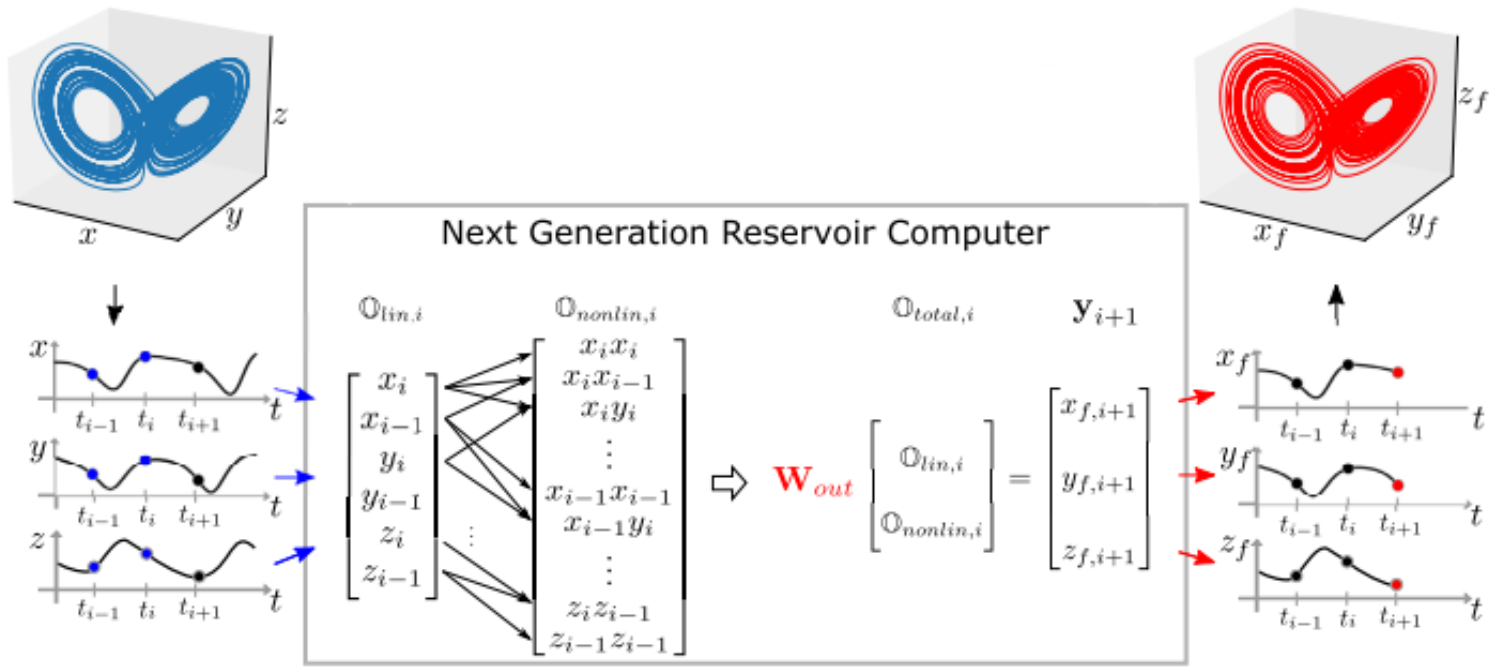

Figure 3: Nouvelle génération du réservoir computing

Comme illustre la figure l'auteur a défini une transformation non linéaire qui nous donne $\boldsymbol{O}_{\text {nonlin, } \boldsymbol{i}}$, à partir des données d'entrée $\boldsymbol{O}_{l i n, i}$. Après il a combiné les deux vecteurs $\boldsymbol{O}_{n o n l i n, i}$ et $\boldsymbol{O}_{\text {lin, }, i}$ ensemble pour obtenir un large vecteur $\boldsymbol{O}_{\text {total }}$ comme suit :

Avec $C$ est une constante

$$
\mathbb{O}_{\text {total }}=c \oplus \mathbb{O}_{\text {lin }} \oplus \mathbb{O}_{\text {nonlin }}
$$

Et comme le $\boldsymbol{R} \boldsymbol{C}$ traditionnel, nous entrainons $\boldsymbol{W}_{\text {out }}$ pour avoir la prédiction $\quad \boldsymbol{Y}_{\text {i+1 }}=\boldsymbol{W}_{\text {out }} \quad \boldsymbol{O}_{\text {total }}$.

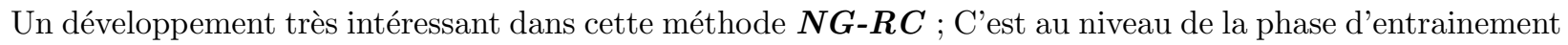
qui maintenant nécessite qu'un peu de data, et dans effectue dans une période très courte en comparaison avec la méthode traditionnelle. Cela très important dans les cas où il est difficile d'obtenir les données.

L'auteur a prouvé aussi que les polynômes d'ordre $\boldsymbol{P}$ peuvent approximer la méthode $\boldsymbol{N} \boldsymbol{V A R}$ en tant qu'un modèle simple du $\boldsymbol{R} \boldsymbol{C}$. Ainsi l'utilisation d'un ordre $\boldsymbol{P}$ bas est largement suffisante pour avoir de bons résultats.

Dans cette partie nous allons présenter les expériences qui prouvent l'efficacité du $\boldsymbol{N G}-\boldsymbol{R} \boldsymbol{C}$, et sa performance sur les trois benchmarking problèmes qui présentent des défis pour le $\boldsymbol{R} \boldsymbol{C}$.

\section{Cas 1: Prévision de la dynamique à court terme}

Exemple: Un modèle simplifié d'un système météorologique 

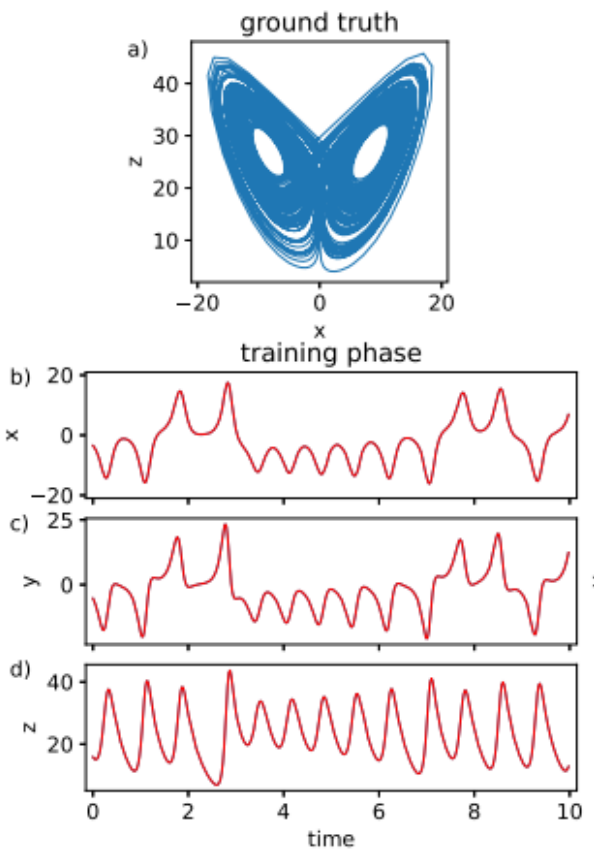

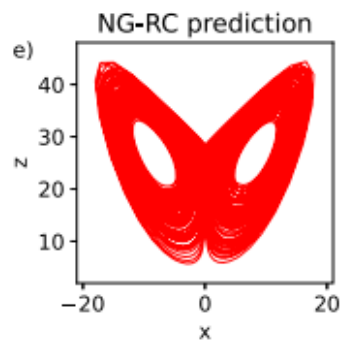

testing phase
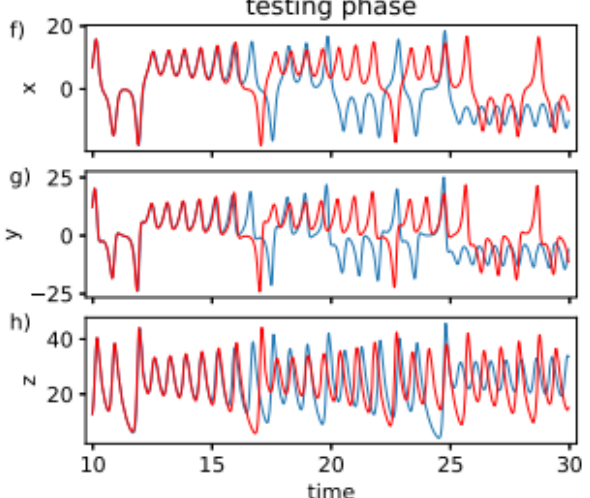

Figure 4: Résultat cas 1

Cas 2: La reproduction du climat à long terme d'un système chaotique

Exemple: La dynamique d'un circuit électronique à double rouleau 

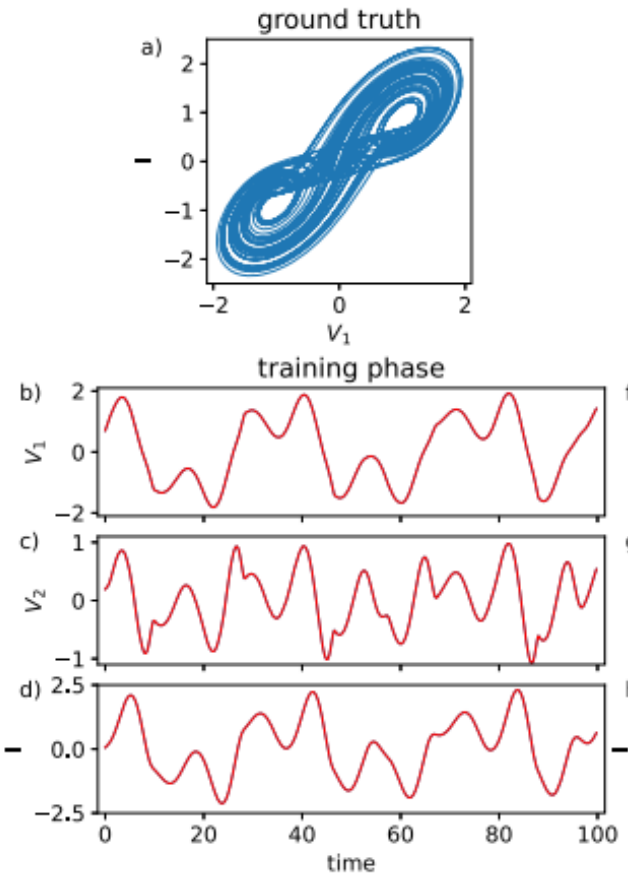
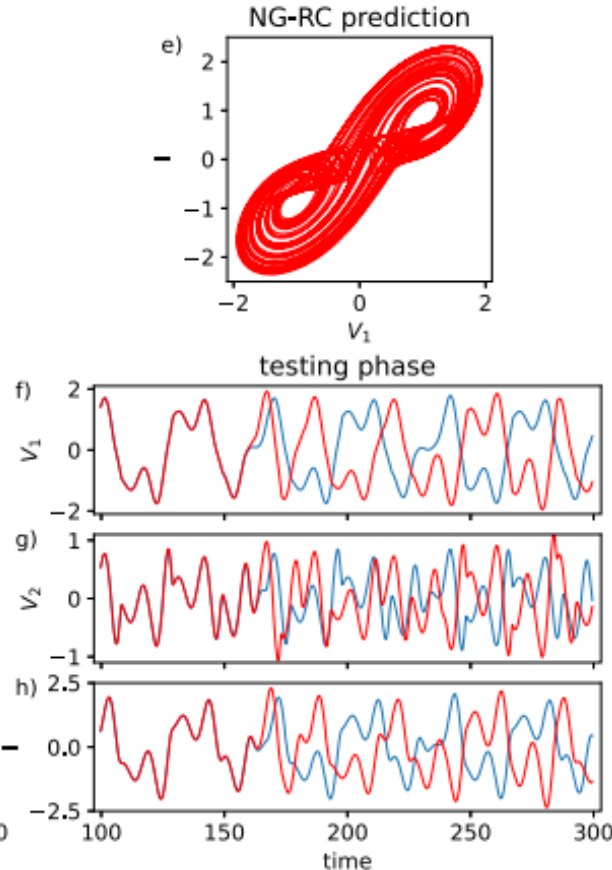

testing phase
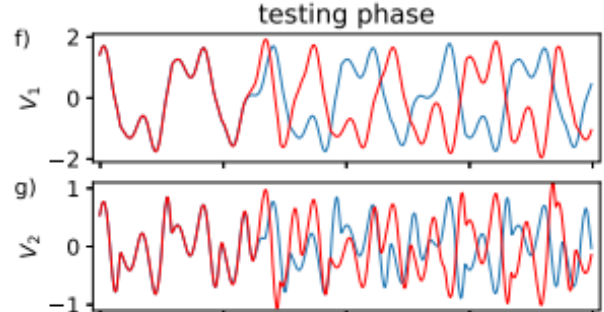

Figure 5: Résultat cas 2

\section{Cas 3: L'inférence du comportement de donnée non observé d'un système dyna- mique}

Exemple: Dans ce 3e cas nous fournissons au $N G-R C$ les trois variables du système de Lorenz63 - cas1pendant l'entraînement, dans le but de déduire la prédiction d'une des variables à partir des autres. Pendant le test, nous ne lui fournissons que les variables x et y et nous déduisons la variable $\mathrm{z}$. 

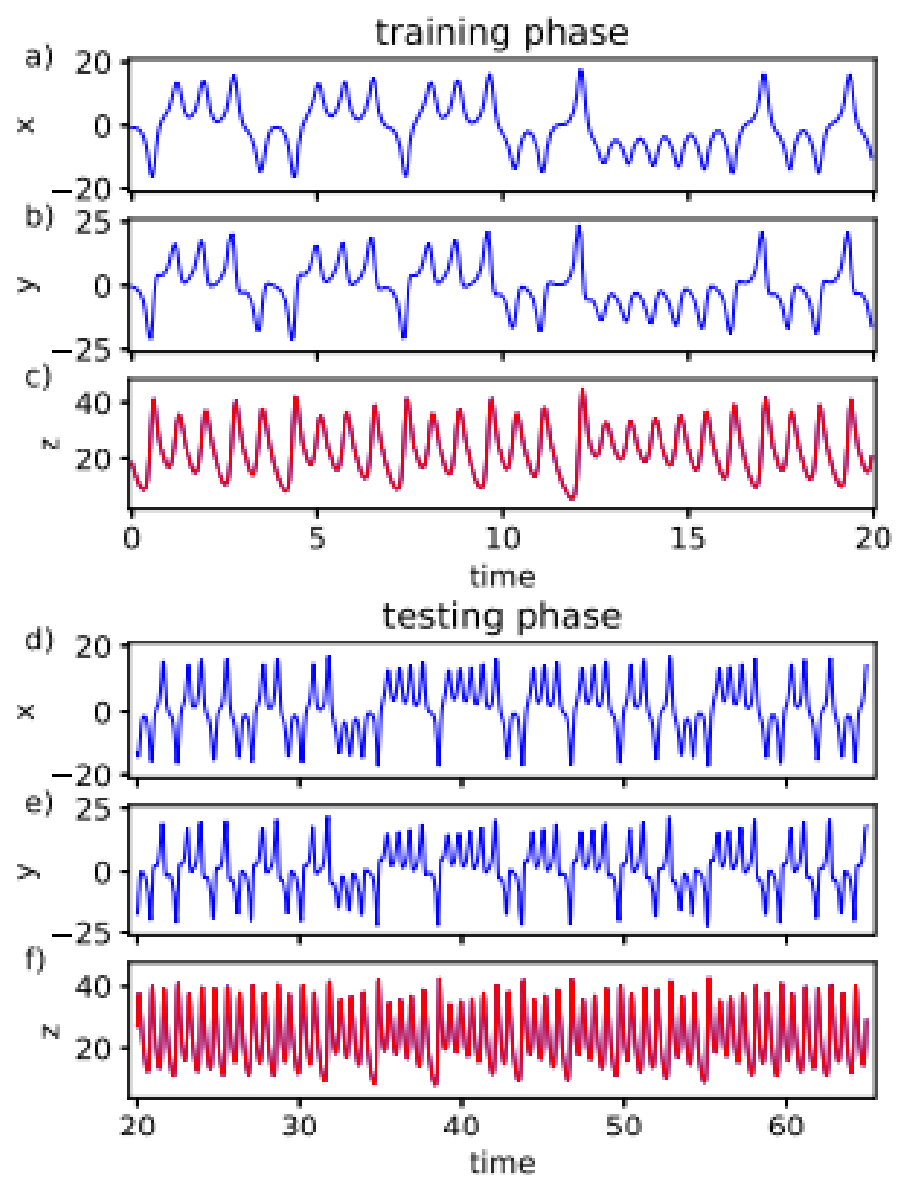

Figure 6: Résultat cas 3

On observe que dans les trois cas le $\boldsymbol{N} \boldsymbol{G}-\boldsymbol{R} \boldsymbol{C}$ ont donné des résultats performants. Pour les cas 1 et 2 l'ensemble des données réel est très similaire de celui qui est prédit. Pour le 3e cas, la prédiction du 3e donnée à partir des deux autres a donné des bonnes performances, les données réelles de $\boldsymbol{Z}$ sont identiques aux prédites. Ces résultats sont obtenus après un temps d'entrainement très court par rapport à un RC traditionnel.

\section{Résultats :}

- Le $\boldsymbol{N G - R C}$ est plus rapide en calcul

- La taille du vecteur caractéristique est beaucoup plus petite

- Moins de métaparamètres à optimiser

- La taille d'ensemble de données d'entrainement est réduite, aussi le temps est plus court

- Moins couteux et donne des résultats performants 


\section{Conclusion}

Les auteurs de cet article ont développé une nouvelle approche et génération du réservoir comptuting qui nous dispense du réseau de neurones avec ses métaparamètres à optimiser, et nous faisons gagner en matière de temps et de data tout en gardant des bonnes performances. Sauf que dans cette méthode, l'utilisateur va aussi définir la transformation non linéaire. Les auteurs ont dit qu'il y a une grande flexibilité dans ce choix, mais il y a toujours une possibilité de choisir une mauvaise. Nous devons donc savoir à quel point ce choix est flexible. Cependant, en constatant les autres résultats sur les 3 problémes de référence du RC en ce qui concerne le temps et données, nous ne pouvons dire qu'ils ont fait une contribution importante dans le domaine du Machine learning.

\section{Références}

Gauthier, D.J., Bollt, E., Griffith, A. et al. Next generation reservoir computing. Nat Commun 12, 5564 (2021). https://doi.org/10.1038/s41467-021-25801-2

Des liens Youtube qui aident à comprendre:

Les réseaux de neurones | Intelligence artificielle 41 - YouTube

La rétropropagation (backprop) | Intelligence artificielle 43 - YouTube 